\title{
Le pathos dans le discours journalistique en Turquie, vis-à-vis des migrants syriens*
}

\section{The Pathos in Turkey's Journalistic Discourse Toward Syrian Migrants}

\author{
Duygu ÖZTIN PASSERAT
}

*Ce travail a été développé à partir de la communication présentée au colloque I'immigration qui a eu lieu à Lisbonne (Portugal) les 26-28 juin 2018.

${ }^{1}$ Prof. Dr., Dokuz Eylül University, Buca Faculty of Education, İzmir, Turkey

ORCID: D.Ö.P. 0000-0002-8695-0984

Corresponding author: Duygu ÖZTIN PASSERAT, Dokuz Eylül Üniversitesi, Buca Eğitim Fakültesi, İzmir, Türkiye

E-mail: doztin@yahoo.fr

Submitted: 30.09 .2020

Revision Requested: 27.11.2020

Last Revision Received: 30.11.2020

Accepted: 01.12.2020

Citation: Oztin Passerat, D. (2020). Le pathos dans le discours journalistique en Turquie, vis-à-vis des migrants syriens. Litera, 30(2), 521-542.

https://doi.org/10.26650/LITERA2020-802707

\section{RÉSUMÉ}

A la suite de la guerre civile éclatée en 2011 en Syrie, la Turquie a accueilli jusqu'à présent plus de 3,5 millions de Syriens. Au début de cette migration, les Syriens étaient bien reçus/perçus, car le peuple turc croyait que les Syriens allaient rentrer dans leur pays à la fin de la guerre civile. Malheureusement cette guerre perdure et les Syriens ne sont pas retournés dans leur pays avec en plus le nombre de Syriens qui atteint maintenant les 3, 609842 millions de personnes. Entre temps le peuple turc confronté à des problèmes socio-économiques grandissants tels que le chômage, l'inflation et l'arabisation des enseignes dans les rues du pays, comment à développer des sentiments et des actes nettement plus xénophobes et racistes envers les syriens. Les médias ont un rôle important dans la construction de ce discours. L'objectif de ce travail est d'analyser ce discours. Pour ce faire, nous allons analyser quelques dépêches et titres choisis aléatoirement entre 2014 et 2018 des journaux nationaux et régionaux. Nous allons répondre aux questions : «Comment les Syriens sont-ils représentés dans les journaux ? ", "Quel est le rôle du discours médiatique et journalistique dans la construction de l'éthos positif et/ou négatif des Syriens ? », « Comment le discours journalistique manipule-t-il le lecteur? ». Mots-clés: Discours journalistique, pathos, éthos, manipulation, migration

\section{ABSTRACT}

Following the civil war that broke out in 2011 in Syria, Turkey has so far welcomed more than 3.5 million Syrians. At the beginning of this migration, Syrians were perceived favorably, because the Turkish people believed that Syrians would go back to their country at the end of the civil war. Unfortunately, this war continues and the Syrians, now numbering 3,609 842 million, have not returned to their country. Meanwhile, the Turkish people face growing socioeconomic problems such as unemployment, inflation, and the Arabization of signs on the streets of the country, creating much more xenophobic and racist feelings and acts toward Syrians. The media has played an important role in the construction of this discourse and this work aims to analyze this discourse. To do this, we will analyze some randomly selected news reports and headlines published between 2014 and 2018 from national and regional newspapers. We will answer the questions: "How are Syrians represented in the newspapers?" "What is the role of media and journalistic discourse in building the positive and/or negative ethos of Syrians?," and "How does journalistic discourse influence the reader?"

Keywords: Journalistic discourse, pathos, ethos, Syrians, manipulation, migration 


\section{EXTENDED ABSTRACT}

Following the civil war that broke out in 2011 in Syria, Turkey has so far welcomed more than 3.5 million Syrians. At the beginning of this migration, Syrians were perceived favorably because Turkish people believed that the Syrians would go back to their country at the end of the civil war. Unfortunately, this war continues and the Syrians, now numbering 3.6 million, have not returned to their country. Since 2014, the Turkish people were confronted with growing socioeconomic problems such as unemployment, inflation and the Arabization of the signs on the streets of the country, which triggered the emergence of a much more xenophobic and racist discourse toward Syrians. The media played an important role in the construction of this discourse. Disseminating information, the ultimate goal of the print media, is a discourse in action and the construction of meaning in the information discourse encompasses a dual process: transformational and transactional processes. In the information process, the context, characterization, storytelling and argumentation of the events play an essential role. This is why the lexicon used in the context or definition of Syrians also shows the point of view of the speaker and the enunciator (or speaker/enunciator) and plays a triggering role in the receiver/reader. This is why there are various appointments of Syrians including archetypes such as guest, migrant, Syrian, refugee etc. Government political actors label them as guests to mitigate the reaction of the Turkish people toward the Syrians to appeal to the feelings of hospitality of the Turks, while the Turkish people call them Syrians (derogatory in this case) or refugees to denigrate and humiliate them.

Moreover, the Syrians, Turkey's scapegoat, are being used by the government in foreign policy and by the opposition in domestic policy to criticize the government, which is responsible for having conducted this Syrian policy. In this study, we will analyze the role of the media in building this hostile discourse, sometimes resulting in hatred toward Syrians. To do this, we will analyze some randomly selected dispatches and headlines. We will address the questions: "How are Syrians represented in the newspapers?", "What is the role of media and journalistic discourse in building the positive and/or negative ethos of Syrians?" and "How does journalistic discourse influence the reader?"

The analysis shows that the media, and especially the print media, aware of the emotions and negative perception of Syrians among the Turkish people, build a discourse based on misinformation to be able to influence readers and subsequently provoke 
racist and xenophobic behaviors toward Syrians. In doing this, it gives importance to legibility, visibility, and dramatization as perfectly defined/described by Charaudeau but it transgresses the stage of intelligibility, the one which, according to Charaudeau, aims to explain events and cases. 


\section{Introduction}

Après la guerre civile syrienne qui a débuté en 2011, la Turquie a accueilli jusqu'à présent, 3,609 842 millions de Syriens. Au début de ce mouvement migratoire, la présence de ces personnes était bien perçue, mais depuis 2014, un discours xénophobe a fait son apparition et a été construit autour des Syriens. Les média et les réseaux sociaux ont joué un rôle très important dans la construction de ce discours, que l'on peut qualifier de discours de haine. Comment les médias ont-elles construit ce discours? Comment le discours médiatique se sert-il des émotions pour persuader son lecteur, mais surtout pour faire naître en lui, la haine contre les Syriens ? Et quels sont les procédés discursifs et énonciatifs qui servent de fondement à ce discours ? Ce sont les questions auxquelles je vais essayer de répondre dans ce travail. Le corpus de mon travail est constitué des dépêches et des titres de divers quotidiens et journaux tant locaux que nationaux publiés entre 2014 et 2018 choisis d'une manière aléatoire.

Pourquoi ce travail ? Premièrement, la Turquie est en train de vivre, depuis 2011, sa première expérience d'immigration proprement dite. Deuxièmement, le discours de haine et de ressentiment qui se développe vis-à-vis des Syriens est en train de préoccuper non seulement le gouvernement mais aussi certains citoyens turcs dont le nombre ne cesse de croître. Troisièmement pour une raison tout à fait subjective, en tant que turque spécialiste de l'analyse du discours, cette xénophobie envers les Syriens, issue du discours médiatique en Turquie, m'interpelle !

\section{La subjectivité dans le discours journalistique}

L'objectif ultime de la presse écrite ou du discours journalistique est d'informer la plus grande masse. L'information est un discours en action. Car « il s'agit là du langage en tant qu'acte de discours, qui témoigne de la manière dont s'organise la circulation de la parole dans une communauté sociale pour en produire du sens » (Charaudeau, 2005, p. 24). D'autre part, la construction du sens dans le discours d'information englobe un double processus : processus de transformation et processus de transaction.

Le processus de transformation consiste à transformer le monde "à signifier » en monde «signifié » en le structurant selon un certain nombre de catégories qui sont elles-mêmes exprimées par des formes. Il s'agit des catégories qui identifient les êtres du monde en les nommant, qui assignent à ces êtres des propriétés en les qualifiant, 
qui décrivent les actions dans lesquelles les êtres sont engagés en narrant, qui fournissent les motifs de ces actions en argumentant, qui évaluent ces êtres, ces propriétés, ces actions et ces motifs en modalisant. L'acte d'informer s'inscrit donc dans ce processus en tant qu'il doit décrire, (identifier-qualifier des faits), raconter (rapporter des événements) et expliquer (fournir les causes de ces faits et événements) (Charaudeau, 2005, p. 30).

Charaudeau illustre le circuit communicationnel d'information comme suit :

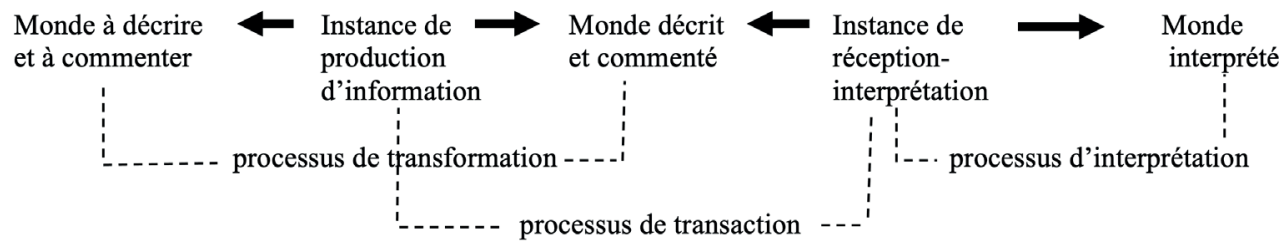

Figure.1

Comme la Figure 1 l'illustre, le discours d'information est d'abord un discours de communication : L'interprétation du monde présuppose l'établissement d'une relation de communication. Autrement dit, c'est le processus de transaction qui dirige le processus de transformation. Par contre, pendant le processus de transformation, les énonciateurs réagissent des fois de manière subjective et parfois même, essayent de manipuler les lecteurs. C'est pourquoi, le discours médiatique est un lieu où les émotions sont utilisées pour persuader l'autre.

\section{I.1. Le lexique utilisé pour les Syriens (identification)}

L'information est un discours en action. Car « il s'agit là du langage en tant qu'acte de discours, qui témoigne de la manière dont s'organise la circulation de la parole dans une communauté sociale pour en produire du sens » (Charaudeau, 2005, p. 24). D'autre part, la construction du sens dans le discours d'information englobe un double processus : processus de transformation et processus de transaction tous deux jouant un rôle important dans le discours journalistique et médiatique. Dans le processus d'information, la nomination, la qualification, la narration et l'argumentation des événements jouent un rôle essentiel. C'est la raison pour laquelle le lexique utilisé (la nomination) dans la nomination ou la définition des Syriens montre également le point de vue du locuteur et de l'énonciateur et joue un rôle déclencheur chez le récepteur/lecteur. 
Les premiers migrants syriens sont apparus en Turquie au mois d'avril de 2011, date à laquelle la guerre en Syrie a éclaté. Ils ont tout d'abord été qualifiés d'invités (misafir), de réfugiés (mülteci), de demandeurs d'asile (sığınmacı) ou encore d'immigrés (göçmen), voire simplement de «Syriens » (Suriyeliler).

Le mot invité véhicule une image de temporalité (on s'attend à ce qu'un invité s'en aille un jour ou l'autre) est utilisé pour la première fois par le Président de République et, désormais, par le pouvoir. Par contre, les termes, réfugiés, migrants et Syriens véhiculent une signification plutôt péjorative et qui portent les sèmes dysphoriques par rapport au mot l'invité.

Selon Amnesty International, un demandeur d'asile (sığınmacı) « est une personne qui sollicite une protection internationale hors des frontières de son pays, mais qui n'a pas encore été reconnue comme réfugié ${ }^{1}$. Autrement dit, ils attendent que leur reconnaissance soit statuée comme réfugié sur leur demande d'asile. Le droit d'asile est un droit Humain, au titre duquel tout le monde devrait être autorisé à entrer dans un autre pays pour y demander l'asile.

Par contre, un réfugié est une personne qui, en cas de retour dans son pays, craint « avec raison d'être persécuté du fait de sa race, de sa religion, de sa nationalité, de son appartenance à un certain groupe ou de ses opinions politiques $»^{2}$. Autrement dit, les réfugiés ont fui leur pays car ils risquent d'y être victimes de graves atteintes à leurs droits Humains et de persécutions. Leur vie et leur sécurité étaient menacées, à tel point qu'ils avaient le sentiment de n'avoir d'autre choix que de quitter leur pays et de chercher un endroit où ils ne seraient plus en danger car leur propre État ne pouvait pas ou ne voulait pas les protéger. Les réfugiés ont droit à une protection internationale.

Toujours selon Amnesty international, il n'existe pas de définition juridique reconnue au niveau international pour les migrants. Les migrants sont des personnes qui vivent hors de leur pays d'origine mais ne sont ni des demandeurs d'asile ni des réfugiés. Certains migrants quittent leur pays pour travailler, faire des études ou rejoindre des membres de leur famille, par exemple. D’autres y sont incités par la pauvreté, les troubles politiques, la violence des bandes criminelles, les catastrophes naturelles ou d'autres problèmes graves.

1 https://www.amnesty.fr/focus/droit-asile, consulté le $1^{\text {er }}$ juin 2018.

2 https://www.amnesty.fr/focus/refugie, consulté le $1^{\text {er }}$ juin 2018. 
Invité : Personne que l'on invite à un repas, à une cérémonie, à une fête etc... (Dictionnaire Le Larousse).

Tableau 1 : Lexiques utilisés pour les Syriens

\begin{tabular}{|l|l|l|l|l|l|l|l|}
\hline & Temporaire & $\begin{array}{l}\text { Proximité } \\
\text { géographique }\end{array}$ & Euphorique & Dysphorique & Permanence & Énonciateur & Volontaire \\
\hline Réfugié & & & & & + & Discours juridique & + \\
\hline $\begin{array}{l}\text { Demandeur } \\
\text { d'asile }\end{array}$ & + & + & & + & & Média & \\
\hline Invité & + & & + & & & $\begin{array}{l}\text { Acteurs } \\
\text { gouvernementaux }\end{array}$ & + \\
\hline Migrant & & & & & + & Le peuple turc & + \\
\hline Syrien & & + & & + & + & $\begin{array}{l}\text { Le peuple turc } \\
\text { et les acteurs } \\
\text { politiques }\end{array}$ & \\
\hline
\end{tabular}

Le terme le plus utilisé pour désigner les Syriens dans le discours journaliste est le mot «Sığınmacı » (demandeur d'asile). Car ce mot véhicule et encore plus en turc, les sèmes péjoratifs et humiliants par rapport aux termes de migrant, d'invité et de réfugié. Les sèmes divergent entre le demandeur d'asile et le réfugié bien qu'ils soient synonymes est le fait que l'acte de réfugié (politique) est un processus planifié alors que celui de demandeur d'asile ne l'est pas, il est, à terme, dans l'obligation de partir. Le mot réfugié a souvent été utilisé pour les sujets qui cherchent une protection auprès d'un pays pour se protéger après la répression politique qui sévit dans son pays d'origine. Cela donne au mot « réfugié » un sens audacieux et courageux voire opposant.

C'est pourquoi, le mot demandeur d'asile a une signification, du récepteur, plus sémantiquement euphorique que le terme réfugié car il signifie la temporalité. Une autre raison est le suffixe $-\mathrm{Cl}$ dérivant des mots à partir des verbes d'actions, plutôt utilisé pour les noms de profession tels que konuşmaCl (intervenant), satıCl (vendeur). Par ailleurs, la racine du mot sığınma désigne également la vulnérabilité du sujet indiqué et non protégé.

Quant au mot invité, on voit que les acteurs politiques (responsables gouvernementaux critiqués pour leur politique syrienne) I'utilisent généralement pour les Syriens. Deux raisons à cette utilisation :

1. Tout d'abord, il vise à alléger le contenu du concept utilisé : essayer d'atténuer cette tragédie humaine et le flux migratoire ;

2. Ensuite tenter d'atténuer la réaction contre les migrants syriens en atténuant le mot. 
Pourquoi autant de mots ? Premièrement comme l'a affirmé Kerbrat-Orrechioni, « toute unité lexicale est, en un sens, subjective puisque les mots de la langue ne sont jamais que des symboles substitutifs et interprétatifs des choses » (Kerbrat-Orrechioni, 1997, p. 70). C'est pourquoi, le choix de substantifs même, démontre notre subjectivité et notre point de vue par rapport à ce qu'on veut désigner. Le mot invité, utilisé plutôt par les acteurs gouvernementaux veut évoquer premièrement, chez le destinataire, que les Syriens ne vont pas rester longtemps dans le pays et deuxièmement qu'ils veulent ainsi faire appel aux valeurs communes du peuple turc connu pour sa grande hospitalité. De même, le mot demandeur d'asile (sığınmacı) utilisé, évoque plutôt le sentiment et les émotions de pitié envers les Syriens. Par contre, le mot réfugié utilisé évoque plutôt les sentiments dysphoriques des énonciateurs envers les Syriens. Car le mot réfugié donne aux Syriens un statut juridique qui effraie le peuple turc. Dernièrement le mot « Syriens » utilisé seul, évoque une idée d'humiliation pour celui qui l'emploie (énonciateur).

De façon générale, qu'ils soient désignés comme des invités (misafir), des réfugiés (mülteci), des migrants (göçmen), des demandeurs d'asile (sığınmacı), les « Syriens » (Suriyeliler) sont toujours évoqués comme un groupe social monolithique par les journaux puis amalgamés par la population qui, par ignorance et désinformation, préfèrent se voiler des différentes réalités et des différents parcours de vie. Les médias turcs jouent ainsi un rôle important dans l'uniformisation et la criminalisation du groupe des Syriens et la diffusion des discours de haine.

\section{De la lisibilité à la visibilité}

Comment les médias turcs stigmatisent-ils les Syriens et comment procèdent-t-il pour créer ce discours hostile et même de haine vis-à-vis des Syriens ? Sémiologiquement parlant, le discours journalistique est un discours hétérogène : il englobe des images, des couleurs et des écritures de types différents. Pareillement aux autres médias, il est unidimensionnel et comme l'affirme Charaudeau, «il est monolocutif et s'organise sur support spatial » (2005, p. 194). C'est pourquoi l'activité discursive est basée sur la conceptualisation. Étant donné la nature visuelle du discours journalistique, la visibilité va de pair avec la lisibilité. Aussi, le journal tient-il à ce que les annonces, les dépêches et les textes soient visibles. Le journal le fait au travers de la mise en page (Une, colonnes, rubriques, images, etc.) et de la titraille (titres, sous-titres, chapeaux). C'est à travers la mise en page et la titraille que le journal accomplit la fonction phatique (attirer l'attention 
du lecteur), épiphanique (annoncer la nouvelle) et synoptique (guider le lecteur à l'aide des éléments visuels). Pour la dimension lisibilité, le journal se doit de rapporter d'une manière lisible les informations nécessaires. Une autre caractéristique est l'intelligibilité. Le journaliste doit expliquer le pourquoi et le comment de ce qu'il dit. Une dernière exigence exercée implicitement mais moins pertinente est la dramatisation. Cette exigence se manifeste dans les titres, et dans les modes d'écritures.

Par ailleurs, un journal comprend plusieurs types de textes : les dépêches, les brèves, les filets, les chroniques, les éditoriaux etc. Tous ces textes relèvent également de plusieurs types d'énonciations qu'on peut qualifier « d'objectifs, de neutre, de subjectifs ou d'engagés ». Ils sont d'ordre phatique parce qu'ils assurent la communication entre le journaliste et le lecteur ; ils sont d'ordre épiphanique, parce qu'ils annoncent ou résument ce que le lecteur va lire ; ils sont d'ordre synoptique, parce qu'ils guident le lecteur. C'est pourquoi, certains titres d'articles incitent le lecteur à lire l'article. De même certains titres montrent-ils l'aspect subjectif et argumentatif de l'article. Autrement dit, l'éthos des textes se détermine dès la lecture du titre. C'est pourquoi les titres accompagnant les images ont plutôt l'objectif de manipuler le lecteur et non pas de le guider. Voici quelques exemples de dépêches et de titres et des Unes de journaux locaux et nationaux sur les Syriens.

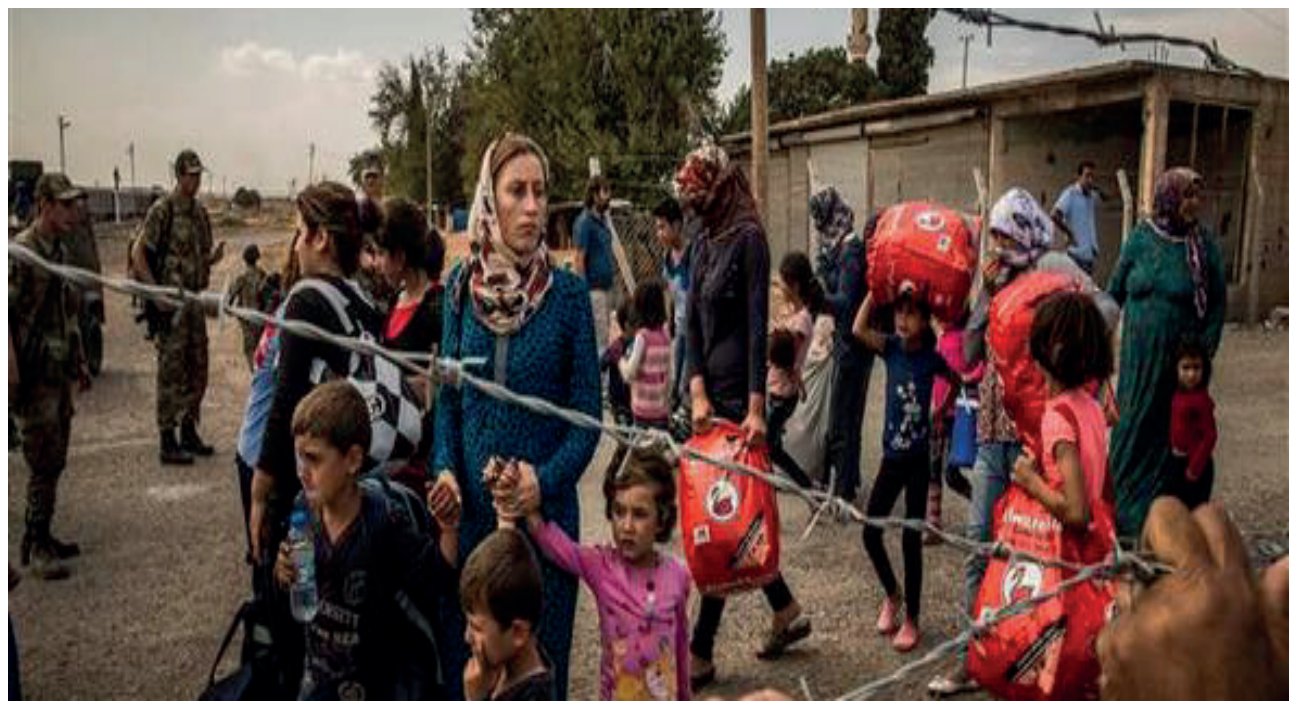

Photo 1 : Hürriyet, le 5 Juillet 2017 


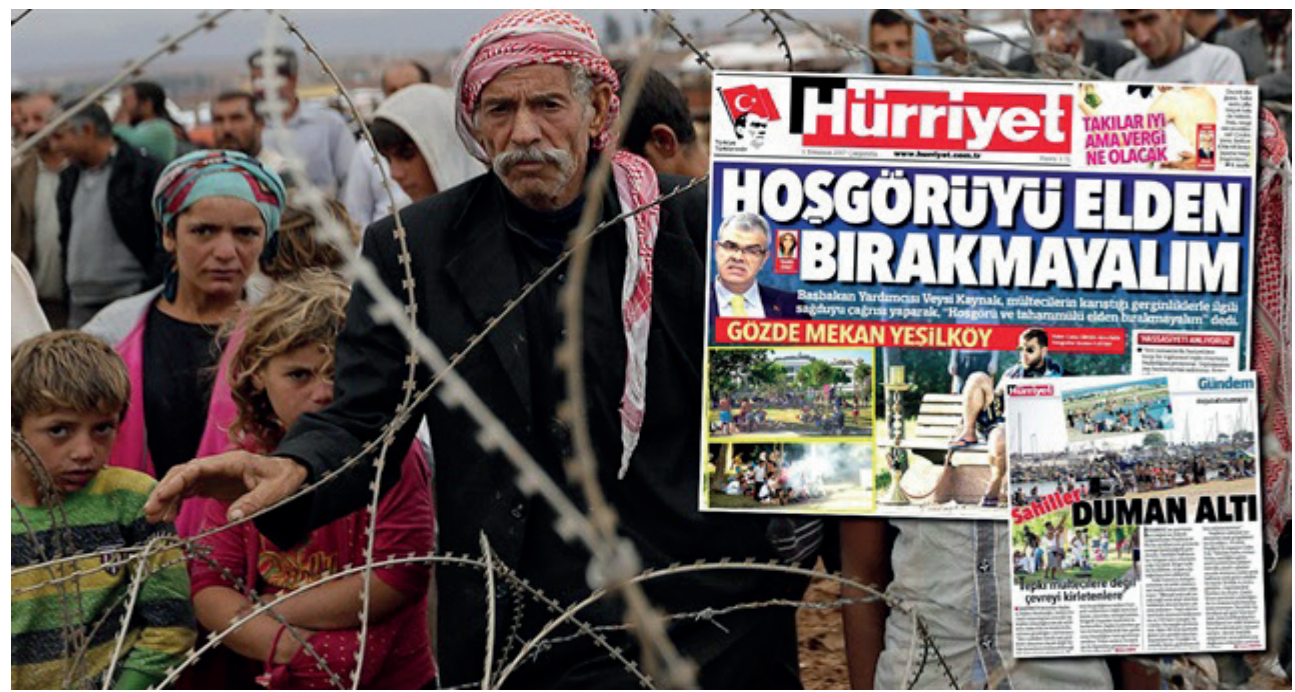

Photo 2 : Hürriyet, le 5 Juillet 2017

Ne laissons pas tomber la tolérance !!!

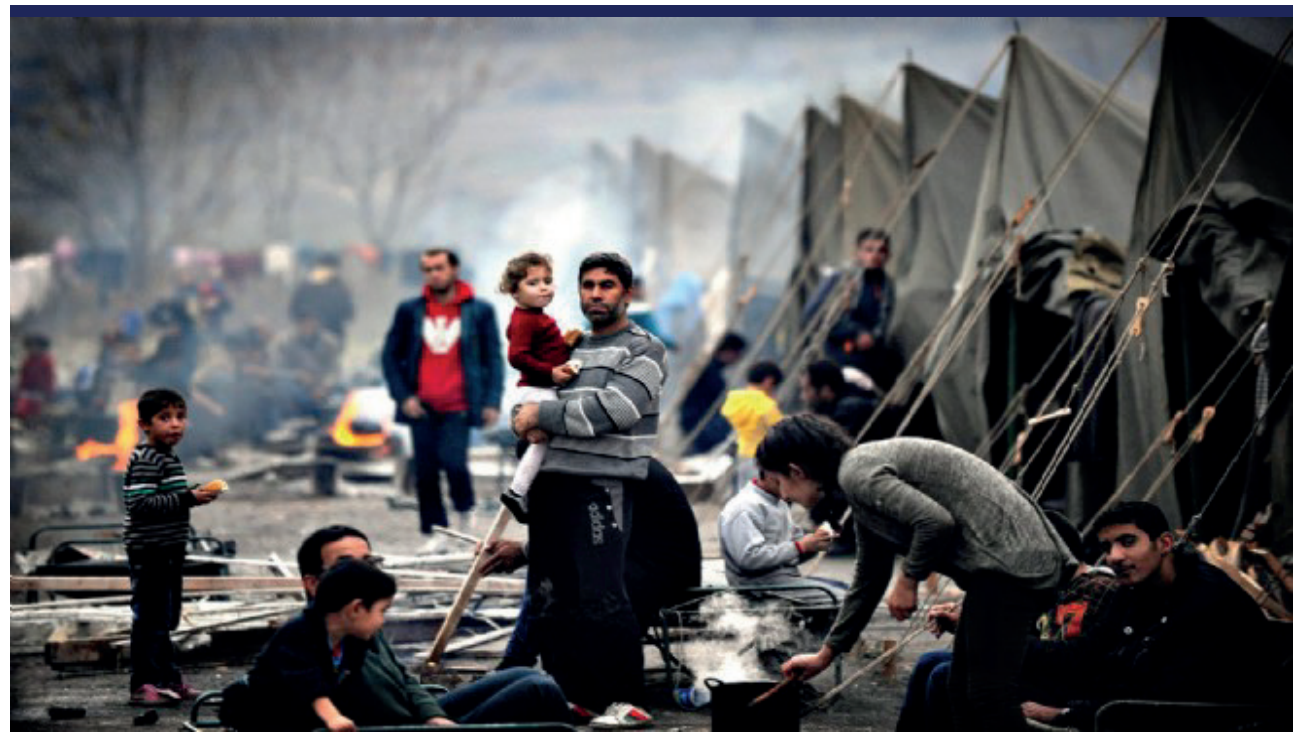

Photo 3 : Hürriyet, le 5 Juillet 2017 


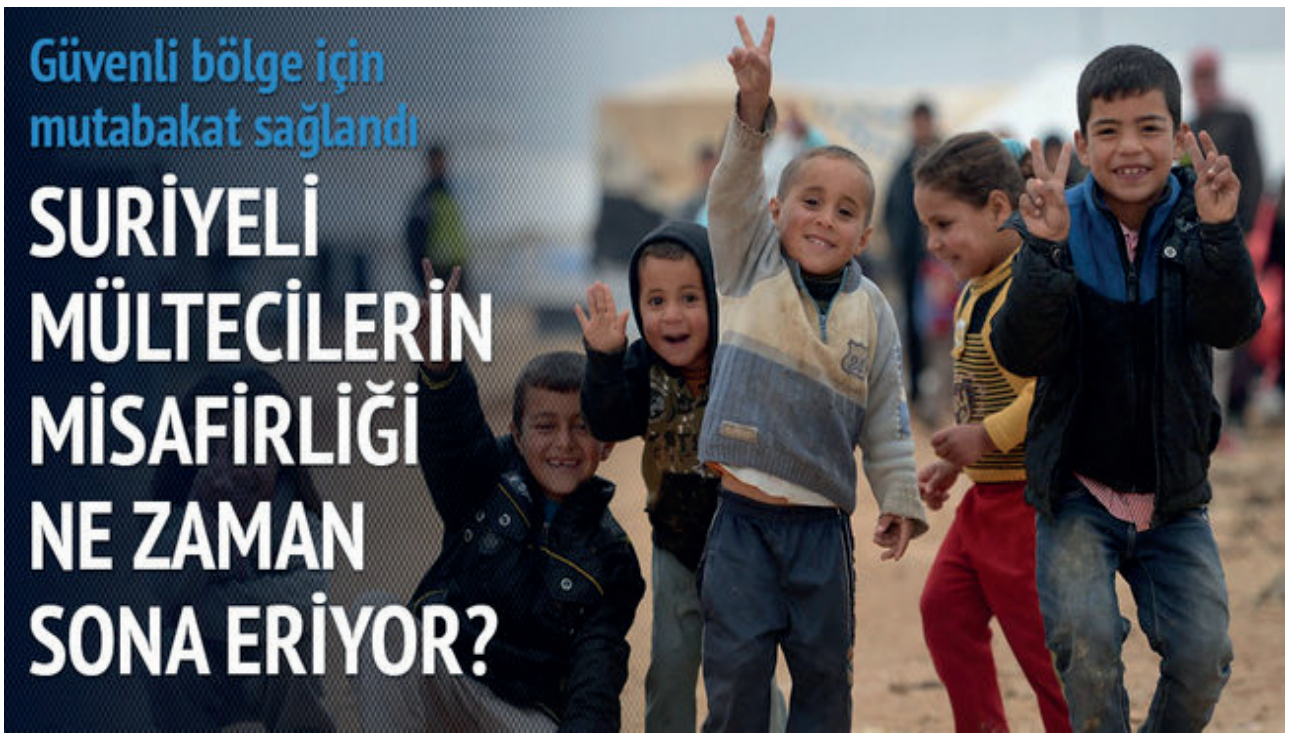

Photo 4 : Sabah, le 1 Juillet 2015

Quand prendra fin l'hospitalité envers les Syriens?

\section{II.1. La construction de l'éthos positif (favorable) des Syriens}

\section{Message iconique (visibilité)}

Les journaux nationaux tels que Hürriyet, Milliyet, Sabah et Cumhuriyet essayent de donner un éthos positif ou à la limite neutre. Ils les représentent comme vulnérables et victimes de la guerre civile en se basant sur le mot demandeur d'asile qui signifie la temporalité. Pour ce faire, dans leur message iconique (visibilité), les journaux font appel à des personnes vulnérables représentées par les femmes, les vieux et les enfants et des conditions de vie précaires montrées par les camps de réfugiés représentés par les tentes de Kızılay (Le Croissant rouge) comme les images ci-dessus l'illustrent. Car, sémiologiquement parlant, les enfants et les femmes sont plus susceptibles de faire naitre des sentiments de pitié et de compassion et par la suite, d'améliorer dans la tête des récepteurs l'éthos vis-à-vis des Syriens. Afin de construire plus d'authenticité et de réalité on voit qu'on a utilisé des images prises dans les camps près de la frontière syrienne avec, en fond, le drapeau turc et protégés par des soldats turcs qui évoquent également l'hospitalité turque (Photo 1, photo 2).

Dans les première et deuxième photos on voit bien en évidence au-devant des photos les barbelés juste devant l'objectif (caméra subjective). Les enfants et un vieil 
homme avec son écharpe locale/traditionnelle, utilisée également par les kurdes de Turquie nous renseigne sur son identité. Aussi pouvons-nous parler d'un langage métonymique. Sous les regards souriants des enfants se cache pourtant un destin peu enviable de pauvreté et de condition de vie misérable. Le fait que les photos 1 et 2 soient prises dans les camps syriens derrière les barbelés souligne l'authenticité et l'objectivité de l'image et augmente l'impression de réalité. Par ailleurs, on associe habituellement ce genre de barbelés aux camps de concentration nazis et cela éveillent un sentiment de pitié chez le lecteur. Dans la troisième photo, on voit un enfant dans les bras d'un homme dans un de ces camps. L'utilisation de la technique de fondu enchainé dans la photo rend la photo poétique et augmente le sentiment de pitié envers les personnages qui figurent sur la photo.

Pour ces 4 photos, on utilise un plan général qui permet à l'énonciateur de déterminer l'espace. Concernant les couleurs, on témoigne de l'utilisation des couleurs sombres qui permettent à l'énonciateur de donner un éthos de vulnérabilité et de misère des Syriens chez le lecteur. De ce fait, nous pouvons dire que ces photos essayent de donner un bon éthos des Syriens en éveillant chez le lecteur la compassion et la pitié.

\section{Message linguistique}

La deuxième et la quatrième photos sont accompagnées par un message linguistique : le premier est un impératif prononcé le 5.07.2017 par le vice premier ministre turc de l'époque. Cet énoncé et ce titre démontre qu'il existe bel et bien un discours d'intolérance envers les Syriens. C'est pourquoi, par cette phrase, l'acteur politique essaye d'apaiser, la colère envers les Syriens en faisant appel à la tolérance de ses compatriotes.

Dans la quatrième photo, on constate cette fois, un énoncé interrogatif « quand prendra fin l'hospitalité envers les Syriens ? " Contrairement au premier message linguistique, celui-ci provoque la peur et le doute chez le lecteur concernant le temps que va durer l'hospitalité envers les Syriens, bien que le message iconique essaye d'éveiller le sentiment de tolérance par l'utilisation de photos d'enfants.

\section{II.2. La construction d'un éthos négatif}

Contrairement à l'éthos de confiance et positif, l'éthos négatif vis-à-vis des Syriens est plutôt représenté dans les colonnes de la troisième page des journaux plutôt régionaux. 
Et pour afficher l'éthos paresseux, violeur et harceleur, on a utilisé des jeunes Syriens barbus et costauds. Ils sont même parfois montrés torses nus. Voici quelques exemples:

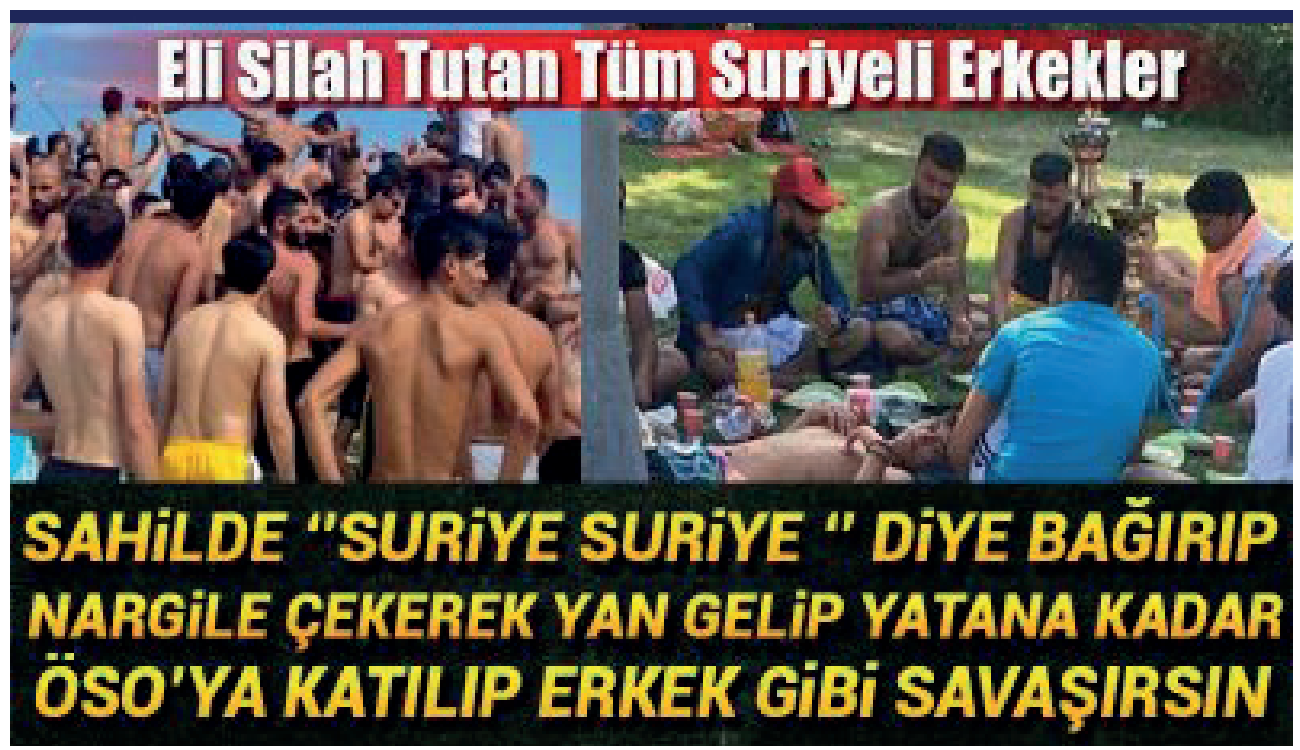

Photo 5 : Kırşehir Haber Türk, le 8 Février 2018

Au lieu de crier «Syrie » à la plage et de fumer de la chicha et t'allonger à la plage, tu peux rejoindre l'armée syrienne comme un brave homme.

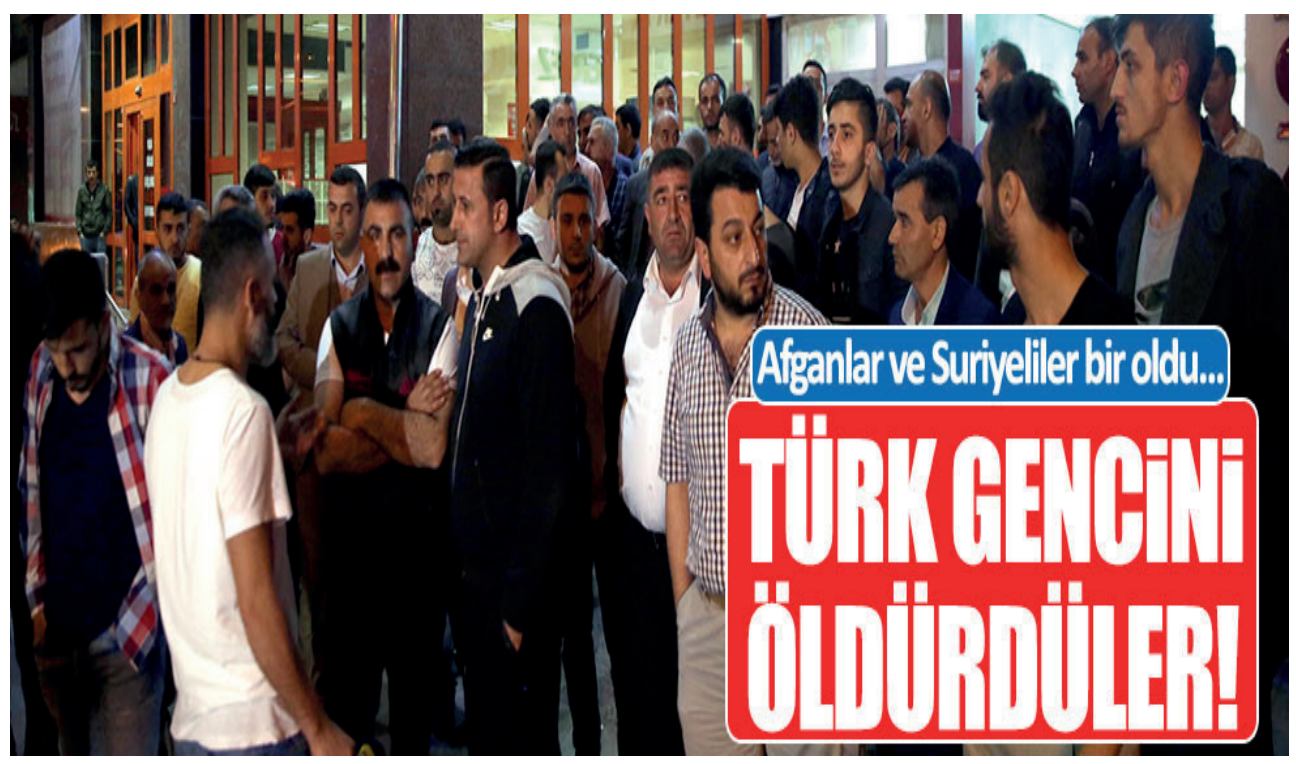

Photo 6 : Yeniçağ, le 15 Mai 2015

Les Afghans et les Syriens ont assassiné un jeune turc. 


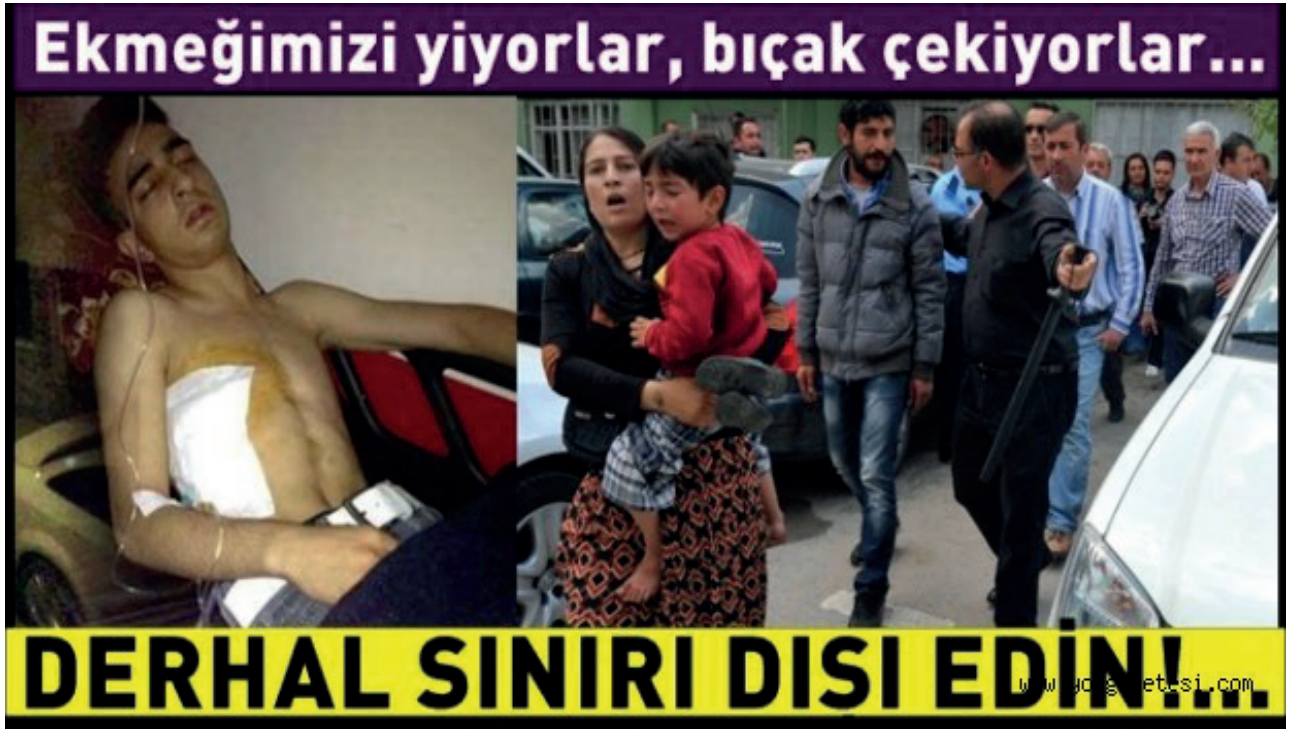

Photo 7 : Yön, le 17 Juillet 2014

Nous, on les nourrit et eux, ils nous poignardent.

EXPULSEZ LES IMMEDIATEMENT !!!

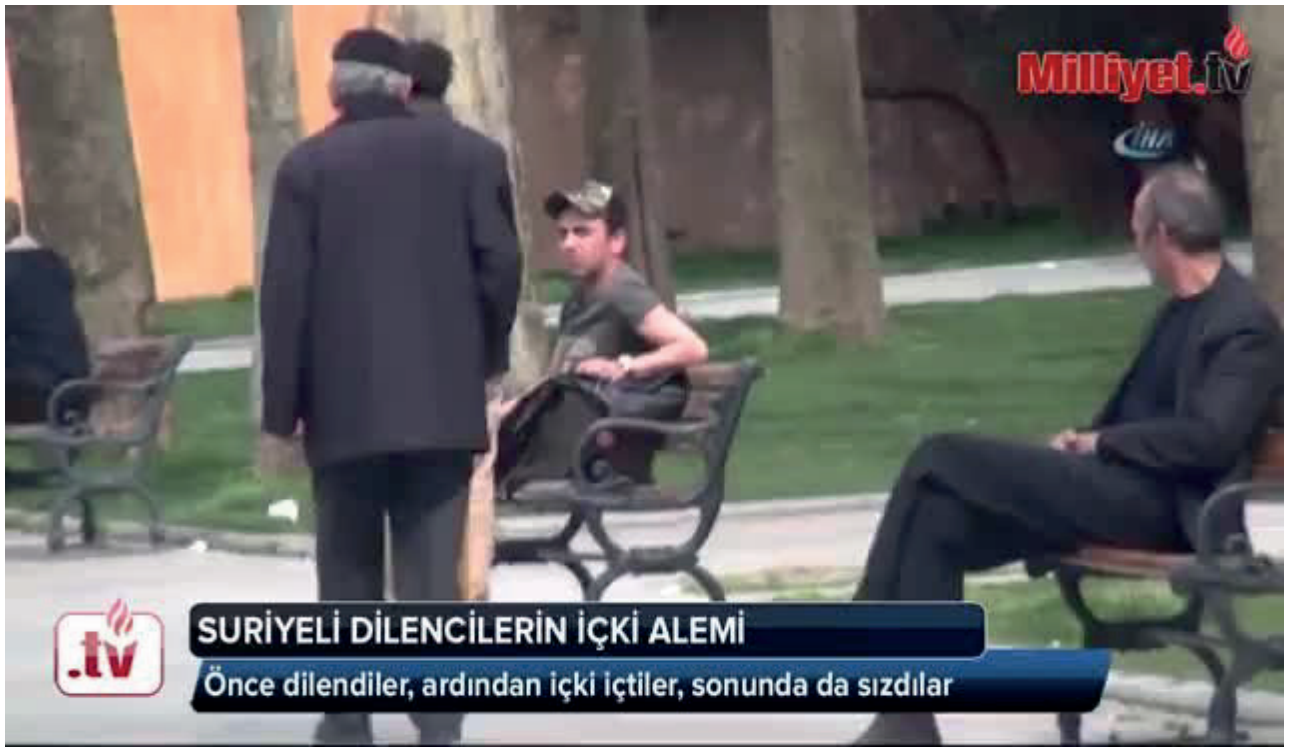

Photo 8 : Milliyet, le 4 Avril 2018

Les mendiants syriens et l'alcool : ils ont d'abord mendié Et puis ils se sont soûlés. 


\section{Message linguistique}

Contrairement au discours neutre et tolérant, pour la construction du discours hostile allant jusqu'à la haine envers les Syriens, on constate que le message linguistique occupe une place importante. Le message linguistique tente non seulement d'expliquer le message iconique mais de le manipuler et de le guider et il joue « le rôle d'ancrage » pour le message iconique comme l'affirme Barthes. (Barthes, 1964) Ainsi, en témoignent les titres et les sous titres de certains journaux qui utilisent un discours hostile envers les Syriens. Ce sont des énoncés fait pour attiser le feu et la colère contre eux. Pour ce faire, on utilise des arguments socio-économiques importants pour le peuple turc : le chômage, les loyers, les vols, etc.

Comme on le constate dans certains titres, il s'agit d'un discours polarisé construit entre eux et nous, les Syriens et les Turcs. La polarisation se trouve dans les choix antonymiques comme nourrir et poignarder dans les mêmes énoncés ou bien dans le choix des verbes dysphoriques comme tuer. Par ailleurs, ce titre « on les nourrit et ils nous poignardent » fait référence à un proverbe : «Qui nourrit un corbeau ne reçoit qu'ordure pour récompense » (Besle kargayı oysun gözünü).

Tableau 2 : Procédés sémioinguistiques utlilisés dans la construction de l'éthos et du pathos

\begin{tabular}{|l|l|l|}
\hline Procédés sémiolinguistiques & L'éthos & Le pathos \\
\hline $\begin{array}{l}\text { Le plan général, la caméra } \\
\text { subjective (on voit les personnes } \\
\text { derrière les barbelés), les femmes, } \\
\text { les enfants et les vieux, les couleurs } \\
\text { sombres et froides. }\end{array}$ & $\begin{array}{l}\text { Les Syriens sont des personnes } \\
\text { vulnérables et victimes de la guerre } \\
\text { civile. }\end{array}$ & La pitié et la compassion \\
\hline $\begin{array}{l}\text { Le plan moyen, les jeunes hommes } \\
\text { barbus et torses nus avec des } \\
\text { colliers en or autour du cou. Les } \\
\text { couleurs frappantes tels que rouge } \\
\text { et noir. Les énoncés linguistiques } \\
\text { (manipulateurs.) }\end{array}$ & $\begin{array}{l}\text { Les Syriens sont des personnes } \\
\text { paresseuses et infidèles à leur pays. }\end{array}$ & La colère, l'indignation, la haine \\
\hline
\end{tabular}

\section{Les Syriens « bouc émissaire » de la Turquie}

Pourquoi ce discours hostile envers les Syriens? Tout d'abord, chaque communauté et chaque culture construit ses propres codes qui influencent la vie affective des individus qui la compose. Et les individus apprennent, dès leur enfance, à catégoriser les acquis comme « bon » ou « mauvais ». La catégorisation entre bon et mauvais est 
non seulement formée par la culture mais également par le système de croyance religieuse dans une même religion. Quelle que soit l'origine des émotions (biologique/ sociale), celles-ci s'expriment, se manifestent en fonction du système de significations dans lequel elles sont construites.

La Turquie n'a jamais eu une l'expérience d'une telle migration, surtout de masse, en provenance d'un pays arabe comme la Syrie. Par ailleurs, l'éthos des Arabes ne présente pas des sèmes favorables/acceptables dans la tête des Turcs. L'éthos prédiscursif ou l'éthos préalable ${ }^{3}$ des Syriens dans la société turque a plutôt des références négatives: ils sont mendiants, ils ne sont pas fidèles à leur pays, ils sont lâches, ce sont des violeurs, ce sont des voleurs, ils ne sont pas propres. C'est pourquoi le stéréotypage concernant les Syriens joue un rôle important dans la construction du discours hostile envers les Syriens.

\section{III.1. L'instrumentalisation des Syriens par l'opposition}

Les principales plaintes de la société turque en référence aux Syriens vont, sans être exhaustives, de la mendicité, du nombre d'enfants, des avantages qu'ils reçoivent de l'État, de la peur d'un phénomène de sunnisation et de l'utilisation de la langue arabe quasi-générale dans certains quartiers (panneaux, enseignes, menus...). C'est pourquoi, ils sont facilement instrumentalisés dans le discours des partis d'opposition en Turquie: le chômage augmente et c'est à cause des Syriens, l'inflation augmente et c'est à cause des Syriens, les crimes augmentent et c'est à cause des Syriens. Le parti qui gouverne le pays est au pouvoir depuis 2003, c'est pourquoi on le considère comme responsable de cette invasion du pays par les Syriens. C'est pourquoi au moment des élections, l'opposition construit son discours pour critiquer la politique syrienne du gouvernement comme l'illustre la dépêche ci-dessous. Aussi pouvons-nous les appeler les boucs émissaires de la Turquie.

3 L'éthos préalable est l'éthos que le locuteur se donne avant son discours. C'est pourquoi nous pouvons le considérer comme la perception ou la réputation du locuteur avant son discours. L'éthos préalable joue un rôle essentiel dans la construction de l'éthos surtout dans le discours politique et médiatique. 


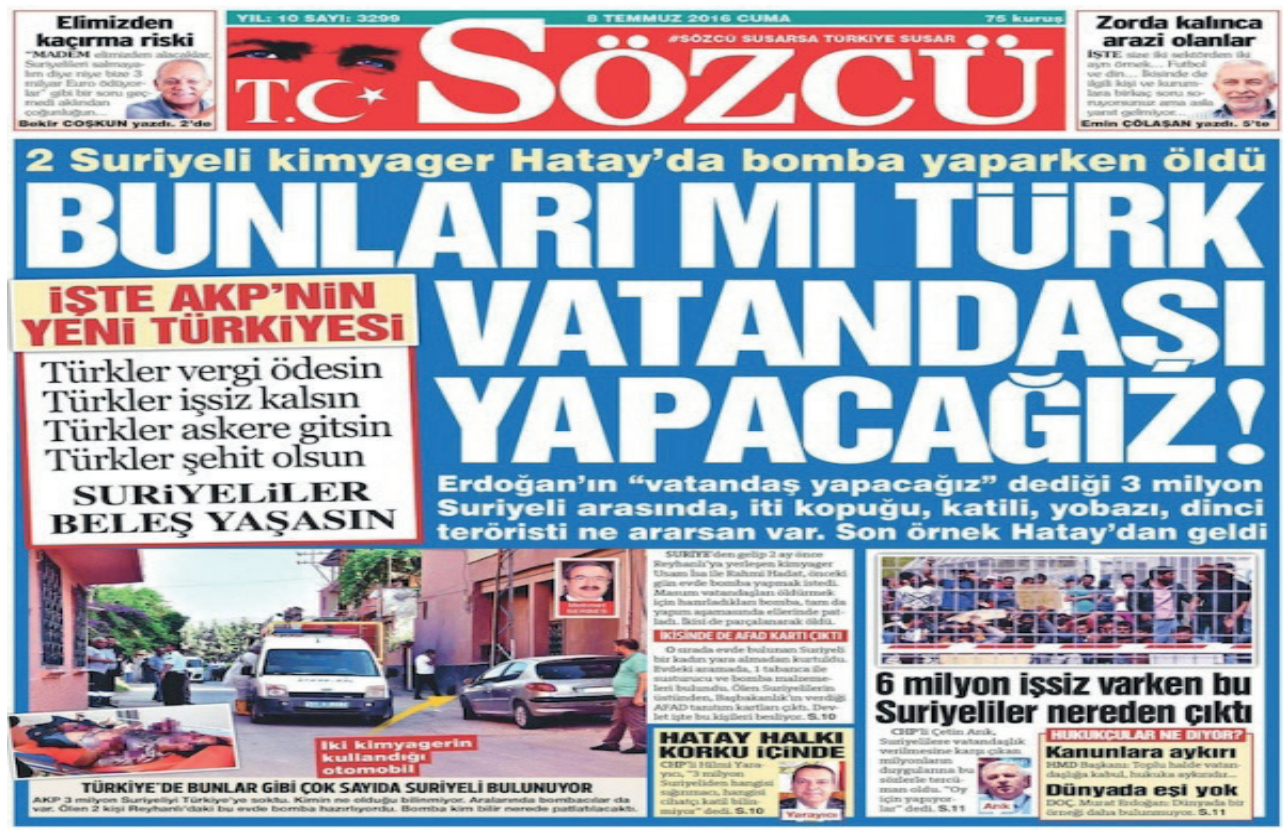

Photo 9 : Sözcü, le 8 Juillet 2016

Deux chimistes syriens morts pendant la fabrication d'une bombe.

C'EST EUX QUI AURONT LA NATIONALITE TURQUE ?

Voici la nouvelle Turquie d'AKP : les Turcs payent les impôts, les Turcs sont au chômage, les Turcs font leur service militaire, les Turcs deviennent martyrs et les Syriens vivent gratuitement

\section{III.2. Les Syriens comme objet de marchandage dans la politique extérieure}

Par ailleurs, le gouvernement actuel instrumentalise les Syriens dans sa politique extérieure afin de marchander avec les pays étrangers et surtout avec l'Europe. Récemment, à la suite de l'attaque de l'armée turque en Syrie au mois de janvier, la Turquie a ouvert les frontières grecques vu que l'Europe a critiqué la Turquie d'avoir attaqué des positions en Syrie et 18.000 syriens ont passés la frontière grecque à la suite de luttes terribles qui ont durés plusieurs semaines.

\section{III.3. La diabolisation (stigmatisation) des Syriens}

Une autre raison de ce discours hostile réside dans la désinformation concernant les Syriens. Voici certaines désinformations (mensonges) sur les Syriens : L'Etat va payer la facture des Syriens, ils seront installés dans les habitats de TOKI, ils vont voter aux élections, ils ne vont pas payer les impôts pour leur voiture, ils vont s'inscrire à l'université 
sans concours etc. Dans les milieux les plus pauvres, certains soulignent que les Syriens constituent une main d'œuvre moins chère, exploitable à souhait, qui bénéficieraient de logements TOKI et de soins gratuits (dû au fait de leur protection temporaire). La classe moyenne hurle à l'injustice quand les jeunes Syriens se voient offrir des places à l'université ou que les services d'urgence sont « envahis par les Syriens alors qu'ils ne payent pas ».

Par ailleurs, la présence massive et hors des camps, des Syriens en Turquie, pour bénéficier d'une certaine liberté de mouvement et de travail, encourage encore davantage ces légendes urbaines. Ainsi, en 2011, on racontait que chaque famille syrienne recevait $1000 \$$ par mois; sur ce sujet, un militant de la plateforme antiraciste Dur De (Dis Stop) s'exprime : « J'ai, une fois, fait le calcul, et si l'État donnait de l'argent à chaque famille... et bien! Ce n'est juste, pas possible ». Aujourd'hui, on entend un peu partout que les jeunes Syriens prennent la place des Turcs dans les universités alors que, comme le rappelle I'hebdomadaire Agos, les bourses universitaires pour les étrangers existent depuis les années 1990 et seuls 1500 étudiants syriens sur 16000 étudiants étrangers au total en bénéficient.

\section{De la manipulation à la provocation (indignation)}

A la suite de cette diabolisation liée à la désinformation et même aux mensonges au sujet des Syriens, il est indispensable que les médias qui se nourrissent des événements scandaleux attisent le feu, et construisent un discours de haine par l'intermédiaire de titres manipulateurs qui éveillent les sentiments d'hostilité et de haine contre les Syriens et provoquent l'indignation des leurs lecteurs. Autrement dit, le processus d'information et de transfert englobant la lisibilité, la visibilité n'a pas respecté l'étape d'intelligibilité qui a pour but d'expliquer le pourquoi des événements. Non seulement cette étape d'intelligibilité a été éludée, mais celle de dramatisation a été poussée a son paroxysme, afin d'éveiller les émotions, dirigée, chez le lecteur. C'est pourquoi il n'est pas étonnant de voir les titres dans les unes des journaux tels que « on ne veut plus des Syriens dans notre ville » qui provoque des sentiments racistes pouvant aller jusqu'à des tentatives de lynchages contre les Syriens (photo 10). Le but principal n'étant plus de donner des informations, mais les "Médias de masse" servent alors et de plus en plus à manipuler et diriger les masses. 


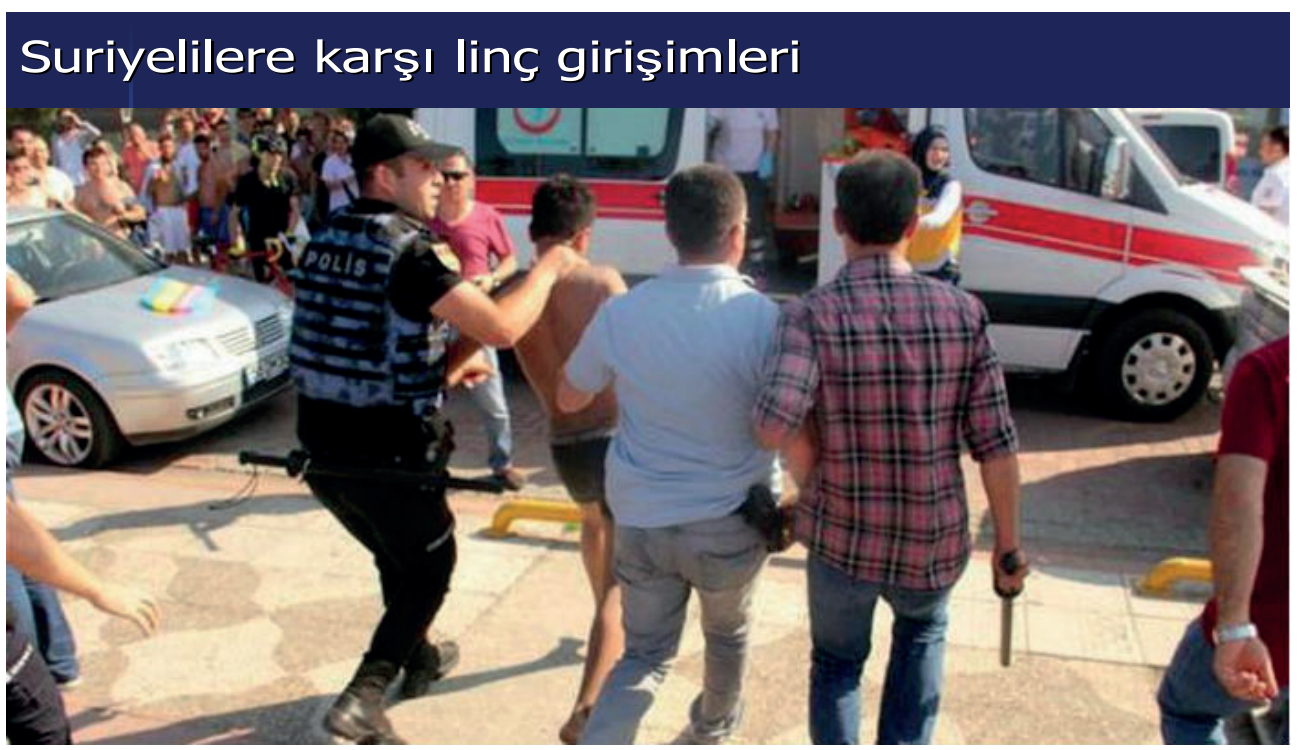

Photo 10 : Yeniçağ, le 2 Juillet 2017 Les tentatives de lynchage contre les Syriens

\section{Conclusion}

Les médias en général jouent un rôle crucial dans le transfert de l'information et dans la perception des événements. Les médias sont tellement puissants qu'on les considère comme un quatrième pouvoir. Déjà en 1840, Balzac affirmait que :"La presse est en France un quatrième pouvoir dans l'État : elle attaque tout et personne ne l'attaque...."4. Par ailleurs, les médias, dans le processus de transfert de l'information, prennent en considération les connaissances, les croyances et les expériences socioculturelles liées aux événements et aux personnes qu'ils vont décrire ou dont ils vont parler puisque l'événement de détection est en quelque sorte le codage de nos expériences précédentes. Pour cette raison, les médias véhiculent des informations en tenant compte des croyances et des contextes de la société dans laquelle ils évoluent. Non seulement ils n'essayent pas de changer les fausses idées et les préjugés, les médias manipulent les gens en déformant, à leur avantage, les vérités sur les événements et sur les personnes. En faisant cela, ils construisent un discours fondé sur la discrimination

4 https://gallica.bnf.fr/ark:/12148/bpt6k1065498g/f103.item, consulté le 10 septembre 2019. 
et sur le pathos et sur les émotions loin de la logique (logos). Ils donnent de l'importance à la visibilité, à la lisibilité mais ils n'expliquent nullement les raisons des événements. Ainsi, l'étape de l'intelligibilité dans le circuit communicationnel dans le discours médiatique est transgressée.

La presse écrite/les journaux qui font partie du discours médiatique fonctionnent de la même façon. Au lieu de donner des informations à la société d'une manière objective, ils jouent sur les émotions et les préjugés sur les Syriens. Si les journaux sont proches du gouvernement, ils essayent d'apaiser la haine et l'hostilité envers les Syriens en faisant cela ils essayent d'éveiller la pitié et la compassion chez le lecteur. Par contre, les journaux régionaux attisent le feu en éveillant chez le lecteur la haine et l'indignation. En faisant cela, ils fondent leurs discours sur la désinformation et ainsi ils manipulent et ils provoquent les gens à s'indigner quitte même à agresser physiquement des Syriens.

Par ailleurs, la désinformation et la manipulation au service de la provocation dans le langage journalistique/médiatique se sert également de l'éthos préalable et négatif des Syriens. Bien que l'on sache que la perception négative des Syriens a de nombreuses raisons, notamment socio-économiques, il est notoire que la presse écrite et les médias jouent un rôle très important dans la construction de cet éthos négatif. En ranimant les émotions de peur et de colère, les médias jouent un rôle pour renforcer ce statut de bouc émissaire au lieu de faciliter leur intégration dans la société turque. A la limite de la désinformation et de la manipulation, ils provoquent des sentiments de haine contre les Syriens.

Les Syriens qu'on les appelle invités ou réfugiés forme une communauté de personnes qui ont fui la guerre dans leur pays et ils sont demandeurs d'asile et a priori, vulnérables. Ils sont en Turquie depuis 9 ans déjà. Ils n'ont aucun statut juridique. Par contre, ils sont, tous les jours de plus en plus, les boucs émissaires de la société turque. Il incombe donc aux chercheurs et aux intellectuels en général de relever la tâche et de remédier à cette désinformation qui inspire la méfiance dans les deux groupes, l'un vis-à-vis de l'autre. Il leur revient surtout de pointer le rôle de l'État: la générosité des discours du Président turc ne doit pas faire oublier qu'en dehors des politiques discursives, aucune politique d'accueil sur le long terme et d'intégration n'a été correctement pensée, ni même anticipée, pour ces millions de personnes vulnérables. 
Évaluation : Évaluation anonyme par des pairs extérieurs.

Conflit d'intérêts : L'auteure n'a aucun conflit d'intérêts à déclarer.

Subvention : L'auteure n'a reçu aucun soutien financier pour ce travail.

Peer-review: Externally peer-reviewed.

Conflict of Interest: The author has no conflict of interest to declare.

Grant Support: The author declared that this study has received no financial support.

\section{Bibliographie}

Amossy, R. (2006). L'argumentation dans le discours. Paris : Armand Colin.

Barthes, R. 1964, Rhétorique de l'image, Communication 4, 44-51. https://www.persee.fr/doc/comm_05888018_1964_num_4_1_1027, consulté le 10 mai 2019.

Charaudeau, P. (2005) Les médias et l'information. Belgique : De Boeck.

Charaudeau, P. (2008). « Pathos et discours politique », M. Rinn (éd), Emotion et Discours, Rennes : Presses Universitaires de Renne, pp.49-58.

Danblon, E. (2005). La fonction persuasive, Anthropologie du discours rhétorique : origines et actualité. Paris : Armand colin.

Declercq, G. (1992). L'art d'argumenter. Paris : Editions Universitaires.

Kerbrat-Orecchioni, C. (1997). L'énonciation, de la subjectivité dans le langage. Paris : Armand Colin.

Michelli, R. (2010). L'émotion argumentée. Paris : Editions du Cerf.

Maingueneau, D. (1999). "Ethos, scénographie, incorporation ", dans Images de soi dans le discours - La construction de l'ethos, éd. Ruth Amossy, Lausanne, Delachaux et Niestlé, p. 75-100.

Maingueneau, D. (2002). "Problèmes d'ethos », Pratiques, 113, p. 55-68.

Perelman, C., Olbrechts-Tyteca, L. (2008). Traité de L'argumentation. Bruxelles : Editions de I'Université de Bruxelles.

Plantin, C., (2011). Les bonnes raisons des émotions. Berne : Peter Lang.

Rinn, M., (2008). Emotions et Discours. Rennes : Presses Universitaires de Rennes.

\section{Bibliographie électronique :}

https://www.liberation.fr/planete/2016/07/03/turquie-erdogan-favorable-a-la-naturalisation-des-refugiessyriens_1463698, consulté le 10 mai 2019.

https://orientxxi.info/magazine/les-refugies-syriens-ebranlent-la-turquie-et-l-union-europeenne,1687, consulté le 10 mai 2019.

https://www.sciencespo.fr/enjeumondial/en/odr/les-refugies-syriens-en-turquie, consulté le 10 mai 2019.

https://m.bianet.org/bianet/toplum/185321-medyada-suriyeli-multeciler-nasil-yer-aliyor, consulté le 10 mai 2019. 
http://uidder.org/yalan_carpitma_ve_hedef_saptirma_isci_kardes_bunlara_inanma.htm, consulté le 10 mai 2019.

https://hrantdink.org/tr/asulis/yayinlar/72-medyada-nefret-soylemi-raporlari/1265-medyada-nefret-soylemive-ayrimci-soylem-2017-raporu, consulté le 10 mai 2019.

http://www.halklarinkoprusu.org/2017/06/halklarin-koprusu-dernegi-turkiye-multeci-raporu/, consulté le 10 mai 2019.

https://hrantdink.org/tr/asulis/faaliyetler/projeler/medyada-nefret-soylemi/934-medyada-suriyeli-multecilereyonelik-nefret-soylemi-videosu-yayinlandi, consulté le 10 mai 2019.

https://www.hurriyet.com.tr/gundem/basbakan-yardimcisi-kaynak-kimsenin-suc-isleme-ozgurluguyoktur-40509701, consulté le 10 mai 2019.

https://www.amnesty.fr, consulté le 10 mai 2019.

https://gallica.bnf.fr/ark:/12148/bpt6k1065498g/f103.item, consulté le 10 mai 2019.

https://www.gazeteler.de/, consulté le 10 mai 2019. 